\title{
Leben und Lebendigkeit - Eine Skizze zum Transzendenzbezug des Natürlichen
}

Hans Weder

Will man im Horizont des Neuen Testaments über die Natur und ihre möglichen tieferen Dimensionen nachdenken, läge es nahe,

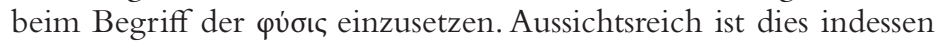
nicht, da der Begriff der Natur im Neuen Testament nur eine marginale und theologisch unergiebige Rolle spielt. Aussichtsreicher ist es, die Mehrdimensionalität des Natürlichen am Beispiel der interessanten Unterscheidung zwischen Leben und Lebendigkeit auszuloten. Diese in der griechischen Sprache mögliche Unterscheidung hat im Neuen Testament eine besondere Ausprägung erhalten.

Die Frage nach dem wahren Leben spielt seit je her in Philosophie und Theologie eine zentrale Rolle. Sie bietet auch eine gute Basis für das interdisziplinäre Gespräch ${ }^{1}$ zwischen Geistes- und Naturwissenschaften, zumal der Begriff des Lebens nicht nur in den Humanities, sondern auch bei vielen Naturwissenschaften im Zentrum des Interesses steht (wie allein schon der Begriff der Lebenswissenschaften deutlich macht). Was das Leben in seinem Wesen sei und wie es gelebt werden kann und soll, gehört noch immer zu den entscheidenden existentiellen Fragen eines jeden Menschen. Es verwundert deshalb nicht, dass die Thematik des Lebens auch im Neuen Testament von grosser Bedeutung ist. Dabei gibt das Neue Testament wichtige Einsichten zu Leben und Lebendigkeit zu entdecken. Im Folgenden soll Einiges davon in Erinnerung gerufen werden.

\section{Aufschlüsse aus dem Sprachgebrauch der grie- chischen Kultur}

Das Griechische hat zwei Wörter für das, wofür im Deutschen nur ein Wort zur Verfügung steht: Sowohl ßios als auch $\zeta \omega \eta \dot{~ w i r d ~}$ in der Regel mit dem deutschen Wort "Leben" wiedergegeben.

\footnotetext{
1 Mit Georg Töpfer, Leben, in: Naturphilosophie. Ein Lehr- und Studienbuch, hg. v. Thomas Kirchhoff u.a., Tübingen 2017, 159: »Leben ist ein Begriff mit einer über einzelne wissenschaftliche Disziplinen hinausweisenden Bedeutung."
} 
Dies könnte auf den ersten Blick den Eindruck erwecken, dass mit diesem einen Wort alles gesagt sei zum Leben. Ein zweiter Blick macht allerdings sofort klar, dass dem überhaupt nicht so ist. "Zwń bezeichnet im Griechischen die physische Lebendigkeit der organischen Wesen, der Tiere und Menschen, aber auch der Pflanzen. $\ll^{2}$ Damit ist das zum Ausdruck gebracht, was heute als Leben im Sinne der Biowissenschaften verstanden wird, die biologische Tatsache des Lebens.

"Es ist konstitutiv für die Biologie, dass sie unter 'Leben das versteht, was allen Lebewesen gemeinsam ist - und dass sie dies terminologisch mit dem Ausdruck Organismus markiert, einem Begriff, der von Bakterien bis zum Menschen anwendbar ist und der bereits einen Ansatzpunkt für die Erklärung der Lebenserscheinungen ausgehend von der inneren Organisation ihrer Körper liefert. $\mathbb{1}^{3}$

Mit dem so gebrauchten Wort "Leben« ist freilich nicht alles gesagt, was zum Leben zu sagen ist. Der übliche Sprachgebrauch versteht unter dem Leben ein weit über das Biologische hinausgehendes Phänomen. Wer Lebenserfahrung hat, hat Einsichten gewonnen, die wenig mit Biologie zu tun haben. Wer von Lebensführung spricht, deutet damit an, dass das Leben - zumindest das menschliche - unter einem ethischen Anspruch steht, dass es verfehlt oder gewonnen werden kann. Leben ist ein mehrdimensionales Phänomen, das sich nicht auf seine biologische Tatsache reduzieren lässt. Umso seltsamer mutet es an, dass die heutige Evolutionsbiologie sich auf eben jene biologische Tatsache beschränkt, um die Entwicklung des Lebens auf unserem Planeten zu beschreiben.

Einen Hinweis auf die Mehrdimensionalität des Lebens gibt das andere griechische Wort, das gewöhnlich ebenfalls mit Leben übersetzt wird, der ßíoc: „Das faktische menschliche Leben erhält immer seine Gestalt in einem individuellen ßioc, in dem es sich gewinnen oder verfehlen kann, und es erwacht die Frage, in welchem ßioc die menschliche $\zeta \omega \eta \dot{~ i n ~ i h r e r ~ E i g e n t l i c h k e i t ~ e r s c h e i n t . ~ « ~ I m ~ U n-~}$ terschied zur physischen Lebendigkeit, die allen Lebewesen - in unterschiedlichen Graden der Intensität - gemeinsam ist, wird mit Bíoc die individuelle Gestalt des Lebens (insbesondere einer menschlichen Person) in den Blick genommen. Neben einer Reihe von anderen Bedeutungen (wie zum Beispiel Lebenszeit, Lebensdauer)

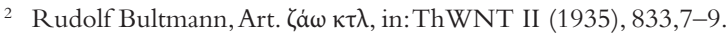

3 Töpfer, Leben, 159.

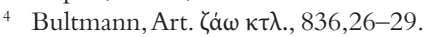


wird das Wort auch zu einem terminus technicus für die "literarische Lebensbeschreibung ${ }^{5}$ (also für das, was wir als Biographie kennen).

Die griechische Sprache hat also die Möglichkeit geschaffen, zwischen Leben und Lebendigkeit zu unterscheiden, ein Unterschied, der insbesondere im Neuen Testament auf interessante Weise zum Vorschein kommt. Während im hermeneutischen Horizont der griechischen Kultur Lebendigkeit ( $\zeta \omega \dot{\eta})$ gleichsam etwas Gegebenes darstellt, etwas, das als solches nicht unter einem ethischen Anspruch steht, verhält es sich beim Leben (ßioc) ganz anders: Für die Gestaltung des Lebens ist es von grundlegender Bedeutung, dass das Leben gewonnen oder verspielt werden kann. Eine individuelle Lebensgestaltung kann gelingen oder misslingen. Unlöslich verbunden mit ihr ist die Frage nach dem gelingenden Leben, die Frage nach dem vollkommenen Leben, das Zeit des Lebens anzustreben ist.

\section{Konturen des neutestamentlichen Sprachge- brauchs}

Schon auf den ersten Blick fällt auf, dass die Verwendung der Wörter $\beta$ ios und $\zeta \omega \eta$ im Neuen Testament stark asymmetrisch ist. Während ßioc lediglich an 10 Stellen vorkommt, ${ }^{6}$ erscheint das Wort $\zeta \omega \eta \dot{~ a n ~}$ über 130 Stellen und beinahe in allen Schriften ${ }^{7}$ des Neuen Testaments. Noch deutlicher ist die Asymmetrie, wenn man die Verben $\beta$ ió $\omega$ und $\zeta \dot{\alpha} \omega$ betrachtet: Das erste kommt ein einziges Mal vor, während für das zweite etwa 140 Stellen gefunden werden. Es überrascht, dass ausgerechnet das Wort, das für die individuelle Lebensführung steht, im Neuen Testament kaum eine Bedeutung hat. An vielen Stellen bedeutet ßioc einfach den "Lebensunterhalt «. ${ }^{8}$ Aus dieser Asymmetrie kann man schliessen, dass das Neue Testament - und das ist überraschend - nicht so sehr um die individuelle Lebensgestaltung besorgt ist. Obwohl die Reflexion des menschlichen Verhaltens im Neuen Testament prominent vertreten ist, gilt die entscheidende Aufmerksamkeit der Lebendigkeit, dem Phänomen

\footnotetext{
5 Ebd. 837,3-5.

${ }^{6}$ Davon einmal erst noch in der Parallele Mk 12,44 par Lk 21,4; sonst nur Belege bei Lk [4x], in 1Tim und 2Tim je 1x und im 1Joh [2x]. Alle andern Schriften brauchen das Wort überhaupt nicht.

7 Einzige Ausnahmen sind die beiden Thessalonicherbriefe und der Philemonbrief.

${ }^{8}$ So zum Beispiel in der Erzählung vom Scherflein der Witwe: "[...] sie aber hat aus ihrem Mangel alles hergegeben, was sie hatte, ihren ganzen Lebensunterhalt.» (Mk 12,44, vgl. Lk 21,4).
} 
also, das man als allgemeine biologische Gegebenheit zu betrachten pflegte. Das Neue Testament konzentriert sich auf die Frage, wie es mit der Lebendigkeit des Menschen steht. Die Frage nach der Qualität der Lebendigkeit scheint zentraler zu sein als die nach der individuellen Lebensführung.

Interessant ist das Gleichnis vom Verlorenen Sohn (Lk 15,1132); dort steht ßíoc für "Vermögen", also für das, was der jüngere Sohn vom Vater für seinen Lebensunterhalt bekommen hat. Dieses Vermögen, das ihn mit seinem Vater verband, verschleudert er in

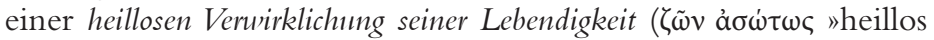
lebend", in einem heillosen Leben). . Seine Lebendigkeit versuchte er dadurch zu steigern, dass er in Saus und Braus lebte. Zwar verspielt er das Leben, den ßíoc, und damit seine Verbindung zum Vater. Die Lebendigkeit aber, die $\zeta \omega \eta$, verliert er dennoch nicht: Als er vor dem Nichts steht, beginnt er, vernünftig nachzudenken; die Verbindung zum Vater ist verloren, die Erinnerung an den Vater aber ist geblieben. Er besinnt sich und macht sich auf den Heimweg. Der Vater empfängt ihn mit offenen Armen; die Umarmung stellt die verlorene Verbindung wieder her. »Feiern müsste man jetzt und sich freuen, « sagt der Vater zum verärgerten Älteren, »denn dieser dein Bruder war tot und ist lebendig geworden ( $\check{(\eta \eta} \sigma \varepsilon v)$, war verloren und ist gefunden worden" (Lk 15,32). Lebendigkeit ist nicht durch den Bios gegeben, der ja verloren war, Lebendigkeit ist gegeben durch das Dasein in der Relation zum Vater.

Diese Konstellation lässt erahnen, wieso für das Neue Testament die Lebendigkeit so stark im Vordergrund steht. Das Leben, das auf einen Lebensunterhalt angewiesen und das die Form der individuellen Lebensführung darstellt, ist nicht zu verwechseln mit der Lebendigkeit, auf die alles ankommt. Lebendigkeit ist nicht schon mit dem Bios gegeben. Dieser ist eine notwendige, nicht aber eine zureichende Bedingung der Lebendigkeit. Den neutestamentlichen Schriften geht es im Kern um eben diese Lebendigkeit; auf vielerlei Weise

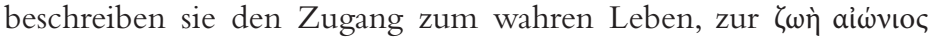
(normalerweise mit "ewiges Leben « übersetzt). Im Unterschied zum allgemein griechischen Sprachgebrauch betrachtet das Neue Testament gerade den ßios als natürliche Gegebenheit, während die $\zeta \omega \eta$, die Lebendigkeit, jederzeit auf dem Spiel steht.

Eingangs hatten wir darauf hingewiesen, dass unterschiedliche Wissenschaften sich im Begriff des Lebens begegnen. Das im Neuen

9 Übersetzt nach der Umschreibung von Wolfgang Harnisch, Die Gleichniserzählungen Jesu. Eine hermeneutische Einführung, Göttingen ${ }^{4}$ 2001, 203. Die Lage des »Betroffenen [wird] in starker Wertung als selbstverschuldet gekennzeichnet [...]«. 
Testament beobachtete Gegenüber von Leben und Lebendigkeit, von biologischer Gegebenheit und zu gewinnender oder zu verlierender Lebendigkeit, soll im Folgenden Anlass dazu sein, ein kleines Stück auf dem Weg des Dialogs zwischen Theologie und Naturwissenschaft zu gehen.

\section{Die Lebendigkeit als Licht für den Menschen}

Sich auf diesen Weg des Dialogs zu machen, erfährt eine nicht geringe Ermutigung durch eine bemerkenswerte Aussage im Johannesprolog. ${ }^{10}$ Vom göttlichen Logos wird dort gesagt, dass in ihm "Lebendigkeit" ( $(\omega \eta \dot{)})$ war, und dass diese Lebendigkeit das Licht war, das jeden Menschen erleuchtet, der zurWelt kommt (Joh 1,4.9). Der Logos, durch den alles Geschaffene qua Schöpfung geprägt ist (Joh 1,3), ist gleichsam der Raum des Lebens, der Lebensraum. In ihm ist das Leben jedoch nicht so, dass er es wie einen Besitz an sich risse, sondern vielmehr so, wie das Leben einst in der Sophia existierte: In ihm ist Lebendigkeit im Modus des Ausgeteilt-Werdens. Der johanneische Christus ist eben nicht nur ein Lehrer, der darüber informiert, wie Lebendigkeit zu bekommen wäre, sondern er teilt die Lebendigkeit aus. Er informiert nicht über das wahre Leben, sondern er macht das Leben derer wahr, die sich ihm anvertrauen ( gen die Erfahrungen mit Jesus eingegangen sein, der auf vielerlei Weise Leben wahr machte und so Lebendigkeit austeilte.

Eben diese im Logos geborgene Lebendigkeit ist es, die nach dem Johannesprolog jeden Menschen erleuchtet, der zur Welt kommt (Joh 1,9). Auch wenn die bei der Geburt geschenkte Lebendigkeit nicht schon den Glanz des wahren Lebens hat, ist sie dennoch ein Fragment jenes Lebens, das der Logos in ganzer Fülle austeilt. Sie ist kein Irrlicht, das den Menschen in die Irre gehen lässt, sondern sie ist wahres Licht, das ihn erleuchtet. ${ }^{11}$ Wird diese Aussage beim Wort genommen, so erhält sie eine schöpfungstheologische Dimension: Die Lebendigkeit, mit welcher der Mensch bei seiner Geburt begabt wird, hat - so ambivalent sie zur Erfahrung kommen mag - eine Offenbarungsdimension: Sie lässt erahnen, wer der Logos ist, der allem

\footnotetext{
${ }^{10}$ Zum Folgenden vgl. Hans Weder, Ursprung im Unvordenklichen. Eine theologische Auslegung des Johannesprologs, Neukirchen-Vluyn 2008,47-50, 55-59.

${ }^{11}$ Der Ton in Joh 1,9 liegt auf der Echtheit dieses Lichts. Vgl Weder, Ursprung im Unvordenklichen, 56.
} 
Geschaffenen das Gepräge gibt. Die biologische Gegebenheit des Lebens verspricht Einblicke in das Wesen des göttlichen Logos.

In eben dieser Aussage des Prologs liegt die Ermächtigung zu einer theologischen Rezeption dessen, was biologisch als Leben verstanden wird. Dies soll im Folgenden als eine Skizze, die eine eingehende Behandlung nicht ersetzen soll, vorgestellt werden.

\section{Metaphorische Dimensionen des Lebens}

Die im Johannesprolog genannte Lebendigkeit der Geburt gleicht einem Tropfen Wasser, der - obwohl winziges Fragment - zum grossen Meer wahrer Lebendigkeit gehört; insofern kann sie das Licht der Menschen sein. Wird die qualitative Differenz zwischen Fragment und Ganzem respektiert, kann das mit der Geburt gegebene Leben nicht direkt theologisch interpretiert werden, da das Ganze nicht einfach als Addition von Fragmenten konzipiert sein kann. Die Sprachform, die jene Zusammengehörigkeit festhält und zugleich die eben genannte Differenz mitsagt, ist die theologische Metapher. Daraus folgt, dass die Merkmale des biologischen Lebens als Fragmente metaphorisch verstanden werden müssen, wenn sie eine Ahnung vom grossen Meer vermitteln sollen. Metaphorisches Verstehen bedeutet hier, die Differenz zur natürlich gegebenen Lebendigkeit nicht zu verwischen und in ihr dennoch den Glanz der wahren Lebendigkeit zu erblicken. Dies soll im Folgenden anhand einiger Merkmale der biologischen Lebendigkeit angedeutet werden.

Ein wichtiges Merkmal des Lebens ist das Wachstum. ${ }^{12}$ Wachstum ist nicht nur ein quantitatives Phänomen; Wachstum führt dazu, dass - inmitten des universellen Trends zu immer grösserer Entropie - immer höhere Komplexität aufgebaut wird. Das Universum mag als Ganzes auf einen Zustand grösstmöglicher Unordnung hinauslaufen, in ihm ereignet sich - mindestens auf einem der Planeten - dennoch Wachstum, Aufbau von immer höherer Ordnung. Theologisch stellt sich die Frage, ob dieses Wachstum sozusagen stumm ist oder ob es ein Versprechen in sich birgt und was dieses Versprechen sein könnte. Auf diese Frage gibt das Gleichnis von der selbstwachsenden Saat (Mk 4,26-29) eine mögliche Antwort: In seinem Zentrum steht die Aussage, dass die Erde von selbst Frucht hervorbringt. In diesem natürlich gegebenen Wachstum entdeckt das Gleichnis das grosse Versprechen des Reiches Gottes: Das Wachstum ist kein Produkt des Menschen, nicht der Mensch bringt die höhere Komplexität der

${ }^{12}$ Vgl.Töpfer, Leben, 161. 
Frucht hervor, sondern die Erde ist es, eine Gegebenheit, die als Fragment das Versprechen der Vollendung der Komplexität, des Reiches Gottes, in sich birgt. Das Wachstum auf dieser Erde ist freilich ambivalent; nicht nur das wogende Kornfeld ist sein Werk, sondern auch der tödliche Tumor. In der metaphorischen Rezeption des Phänomens wird - wie das angesprochene Gleichnis zeigt - alles Negative ausgeblendet, damit das vielversprechende Wachstum aus dem Gemenge von Gut und Böse identifiziert wird und zum Leuchten kommt. Und eben dieses Wachstum wird zum Sprechen gebracht als ein Phänomen der Kreativität, die dem vermuteten Schicksal universeller Entropie entgegensteht.

So sehr das Brot, das die Erde von selbst hervorbringt, zum Leben absolut nötig ist, so sehr ist auch es ambivalent und verderblich. Und dennoch verspricht gerade die Gabe dieses Brotes, dass der Mensch nicht vom Brot allein lebt (wie der matthäische Christus dem Versucher mit den Worten der Heiligen Schrift entgegenhält, vgl. Mt 4,4). Das Brot verspricht, gerade weil es den Hunger des Körpers stillt, eine Nahrung von qualitativ anderer Art, eine Nahrung, die mehr als notwendig zum Leben ist, weil sie die Lebendigkeit steigert. In der metaphorischen Rezeption wird eine Nahrung entdeckt, die nicht von dieser Welt ist und die den Menschen dennoch in dieser Welt ein Mehr an Lebendigkeit zuspricht.

Damit ist ein weiteres fundamentales Merkmal des Lebens ins Blickfeld gerückt, die Ernährung. ${ }^{13}$ Lebende Wesen sind keine geschlossenen Systeme, sie sind vielmehr auf die Zufuhr von Energie angewiesen. Diese Angewiesenheit endet erst mit dem Tod. Man könnte diese Angewiesenheit als Abhängigkeit interpretieren und sie zur Signatur des menschlichen Daseins machen, wie dies in der Neuzeit nicht selten geschehen ist. So verstanden ruft die condition humaine geradezu nach dem Ausgang aus jener Abhängigkeit, sie legt den entschlossenen Schritt in die Unabhängigkeit nahe. Ist dieser Schritt getan, entsteht die Vorstellung des autonomen Menschen, dessen Existenz nur dann eigentlich ist, wenn sie autonom ist. Die Theologie hat seit je mit Recht Argumente gegen dieses Menschenbild vorgebracht, obwohl sie sich mit dem Einspruch gegen das autonome Menschsein grossen Missverständnissen ausgesetzt hat. Obwohl der Einspruch als Plädoyer für die alte Abhängigkeit missverstanden werden konnte, ist er mit Recht geschehen; denn der auf nichts und niemanden angewiesene Mensch ist theologisch gesprochen der homo incurvatus in se, der in der Sünde auf sich selbst bezogene und insofern auf sich selbst zurückgeworfene Mensch.

${ }^{13}$ Vgl. ebd. 
Dieser elementaren Verkehrtheit der Sünde wird er allerdings erst gewahr, wenn er dem vergebenden Gott begegnet ist, dem Christus, der des Menschen Distanz zu Gott überwindet, indem er die Distanziertheit des Menschen am eigenen Leibe erträgt. So wirkungslos die Aufforderung an den Menschen sein mag, sein Ohr dem Hören des göttlichen Wortes zu öffnen, so sehr gibt es das Phänomen, dass ein Wort selbst den Weg zum Innern des Adressaten sucht, indem es - durch seine Attraktivität zum Beispiel - seine Ohren öffnet. So wenig der Mensch in der Lage ist, seine eigene Verschlossenheit zu überwinden, so sehr gibt es ein Externum, das ihn zu öffnen vermag.

Ein weiteres grundlegendes Merkmal des Lebens ist die Bewegung. ${ }^{14}$ Diese Eigenschaft wird schon in der Antike hervorgehoben, wobei der Akzent auf der "Selbstbewegung im Unterschied von mechanischer Bewegung ${ }^{15}$ liegt. In der Tat gehört es beispielsweise zum lebendigen Menschen, dass er sich selbst bewegen kann, wohin er will, wenn er die Möglichkeit und den Bewegungsraum hat. Diese Selbstbewegung gewinnt neben der lokalen auch unterschiedliche geistige oder metaphorische Dimensionen. In der Phantasie bewegt sich der Mensch zu neuen Ufern, in der Hoffnung bewegt sich der Mensch zu einem besseren Leben, in der Ethik bewegt sich der Mensch in Richtung Verbesserung der Welt. Die fundamentale Rolle der Selbstbewegung wird zum Beispiel in der Stoa dadurch unterstrichen, dass die Leidenschaften (die $\pi \dot{a} \theta \eta$ ) in ihre Schranken gewiesen werden. Wo es um die vernünftige Bewegung des Menschen geht, haben die Leidenschaften nichts zu suchen. ${ }^{16}$ Das Richtige tut der kühle Rechner, nicht der leidenschaftliche Engagierte.

Im Unterschied zu diesem die Selbstbewegung ins Zentrum stellenden Verständnis der Leidenschaften fällt auf, dass eine Leidenschaft wie das Mitleid ${ }^{17}$ bei Jesus eine zentrale Rolle spielt, und zwar im Blick auf eine Wende zum Guten. Im Gleichnis vom Verlorenen

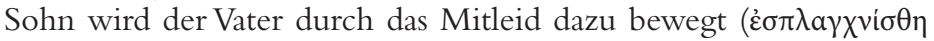
Lk 15,20b), den Sohn zu umarmen und ihn erneut Sohn sein zu lassen, noch bevor dieser sein Sündenbekenntnis ablegen und seine

\footnotetext{
${ }^{14}$ Vgl. ebd. (alternativ dazu nennt Töpfer das Stehen).

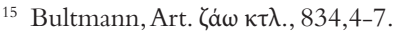

${ }^{16}$ Der Mensch "muss Herr werden über die Einwirkungen, welche sie [die Welt] auf ihn ausübt. Diese Einwirkungen aber bestehen in den Gefühlen und Begehrungen, die Leben und Welt im Menschen erregen, sie sind Störungen seines eigenen Wesens, Leidenszustände ( $\pi \dot{a} \theta \eta$, affectus) «, Wilhelm Windelband, Lehrbuch der Geschichte der Philosophie [1892], hg. v. Heinz Heimsoeth, Tübingen $\left.{ }^{15} 1951,141\right)$.

${ }^{17}$ Zur Verbreitung dieser Leidenschaft im Alten und Neuen Testament vgl. das soeben erschienene Jahrbuch für Biblische Theologie, hg. v. Irmtraud Fischer u.a., Mitleid und Mitleiden, Göttingen 2018,31-182.
} 
Zerknirschung zeigen kann. Das Mitleid ist es, das den Vater zum Vater werden lässt, lange bevor sich die Versuchung seiner bemächtigt, dem Wunsch des Sohnes zu entsprechen und - eine Zeitlang jedenfalls - strenger Arbeitgeber zu werden. In der Geschichte vom barmherzigen Samaritaner (Lk 10,30-35) steht ebenso das Mitleid am entscheidenden Punkt der Wendung zum Guten. Priester und Levit sahen den, der geschunden am Boden lag, und gingen mit guten Gründen vorüber. Auch der Samaritaner sah ihn, auch er hätte gewiss gute Gründe gehabt, ihn liegen zu lassen. Aber im entscheidenden Moment wurde er vom Mitleid bewegt ( $\dot{\varepsilon} \sigma \pi \lambda \alpha \gamma \chi v i \sigma \theta \eta)$. Dieses Mitleid brachte ihn dazu, sich herabzubeugen zu dem, der am Boden lag, ihm aufzuhelfen und für ihn zu sorgen. Das Mitleid war es, das diesen Menschen zum Tun des Guten brachte.

Diese an zwei Beispielen gezeigte Rolle des Mitleids macht aufmerksam auf eine Alternative zu dem sich selbst bewegenden Menschsein..$^{18}$ Diese Leidenschaft macht aufmerksam auf das Phänomen, dass der Mensch nicht nur sich selbst bewegt, sondern dass er - gerade in Richtung des Guten - bewegt wird und diese Bewegung auch fundamental nötig hat. Das Mitleid ist die Leidenschaft, die den Menschen unmittelbar an die Not des andern bindet und die deshalb allem vernünftigen ethischen Begründen zuvorkommt, einem Begründen, das nicht selten dazu führt, dass das notwendige Gute - gewiss aus guten Gründen - gerade nicht geschieht. ${ }^{19}$

Insbesondere in den Briefen des Paulus wird dem Phänomen des Bewegt-Seins grosse Aufmerksamkeit zuteil. Die den Menschen bewegende Kraft heisst hier Geist, $\pi v \varepsilon \tilde{u} \mu \alpha$. Der göttliche Geist ist der Raum, in welchem die Glaubenden leben, er ist die Kraft, welche sie im Einklang mit ihrem Lebensraum leben lässt (Gal 5,25). ${ }^{20}$ Daraus folgt dann, dass die Menschen nicht mehr verpflichtet sind,

\footnotetext{
${ }^{18}$ Der sich selbst bewegende, autonome Mensch, der stets im Besitze seiner Kräfte ist, dürfte auch die treibende Kraft für die stoischen Skepsis gegenüber den Leidenschaften sein, auch gegenüber dem Mitgefühl. Zwar ist die Einstellung der Stoa zu den moralischen Gefühlen differenziert zu betrachten; dennoch ist es erst im Christentum zu einer positiven Bewertung des Mitgefühls gekommen (so Christoph Horn, Mitleid, Parteilichkeit und fürsorgliche Liebe. Einige Beobachtungen zu den Stoikern und Kant, in: Mitleid, hg. v. Fischer u.a., 288-292).

${ }_{19}$ Die im modernen Bewusstsein entstandene pejorative Konnotation (beschrieben ebd., 287f.) des Mitleids (als von oben herab, als untätig, als unverlässlich) führt in die Irre und müsste dringend überwunden werden.

${ }^{20}$ Der Vers stellt das Leben ( $\left.\zeta \tilde{\omega} \mu \varepsilon v\right)$ in der Relation zum Geist (einfacher Dativ) neben das "Im-Einklang-Sein" ( $\sigma \tau(\chi \tilde{\omega} \mu \varepsilon v)$ mit dem Geist. Er zielt auf die Harmonie der Lebensführung mit dem Raum, in welchem das Leben stattfindet und durch welchen es geprägt ist. Daraus eine Kombination von Sein und Sollen, von Indikativ und Imperativ abzuleiten, ist weder philologisch noch theologisch sinnvoll (gegen
} 
"nach dem Fleisch" (Röm 8,12) zu leben. Sie sind nicht mehr darauf beschränkt, ihre Lebendigkeit durch das zu produzieren, was im Bereich des Fleisches, in der Reichweite ihrer Hände und Köpfe ist. Ihre Lebendigkeit verdankt sich nicht mehr allein ihrer Selbstbewegung (obwohl diese gerade auch und unbedingt ihr Schönes hat). Ihre Lebendigkeit wird dadurch gesteigert, dass sie sich dem Geist des Christus aussetzen und so zu Dingen bewegt werden, zu denen sie sich nie hätten bewegen können. Gewiss ist die Bewegung, die von Leidenschaften wie dem Mitleid oder der Trauer ausgeht, nicht dasselbe wie die Bewegung durch den göttlichen Geist. Aber in der metaphorischen Rezeption jener Bewegtheit kann eine Anschauung von der Bewegung durch den kreativen Geist Gottes gewonnen werden. Ihre Tiefe beruht gerade darauf, dass die Bewegung durch den Geist (in der metaphorischen Prädikation) einerseits der Bewegung durch das Mitleid ähnlich ist und andererseits zugleich völlig unähnlich ist.

Als letztes hier zu skizzierendes Merkmal des Lebens soll unser Blick auf die Wahrnehmung fallen. ${ }^{21}$ Während einem Stein aus Granit jede Möglichkeit fehlt, etwas von seiner Welt wahrzunehmen, gehört die auf mannigfache Weise ermöglichte Wahrnehmung elementar zum Leben. Schon in der Antike wird das Erkennen, die $\theta \varepsilon \omega$ pia, als die höchste und für den Menschen charakteristische Möglichkeit verstanden, seine Lebendigkeit zu vollziehen. ${ }^{22}$ Wahrnehmung wiederum ermöglicht es, dass ein Wesen in eine Beziehung zu dem tritt, was nicht es selbst ist - sei es, indem es das Andere wahrnimmt, sei es, dass es seinerseits vom Anderen wahrgenommen wird. Die Phänomene der Wahrnehmung und der Beziehung spielen in der neutestamentlichen Sicht menschlicher Lebendigkeit ebenfalls eine fundamentale Rolle. Als Beispiel sei das Gleichnis vom vielerlei Acker (Mk 4,3-9) genannt: Es wird förmlich in den Rahmen der Wahrnehmung gestellt: "Hört! (Mk 4,3a) steht als Aufforderung vor der Erzählung. "Wer Ohren hat zu hören, der höre« (Mk 4,9), diese auch sonst oft wiederkehrende Formel schliesst sich an die Erzählung an. ${ }^{23}$ Das Gleichnis selbst thematisiert den Aussichtsreichtum des Hörens so, dass es erzählend die Aufmerksamkeit der Angeredeten sucht und ihnen so das Hören gleichsam entlockt.

Jürgen Becker, Der Brief an die Galater, in: Ders., Die Briefe an die Galater, Epheser, Philipper, Kolosser, Thessalonicher und Philemon, Göttingen 1990, 74).

${ }^{21} \mathrm{Vgl}$. Töpfer, Leben, 161.

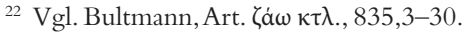

${ }^{23}$ Zum Gleichnis vgl. Hans Weder, Die Gleichnisse Jesu als Metaphern. Traditionsund redaktionsgeschichtliche Analysen und Interpretationen [1978], Göttingen ${ }^{4} 1990$, $108-117$. 
Zur Wahrnehmung hat es stets gehört, dass der Mensch nicht nur seine Welt und seine Mitmenschen wahrnimmt, sondern dass er immer auch Augen für das Unsichtbare hatte, für das, was nicht vor allen Augen liegt, und Ohren für ein schöpferisches Wort, das ihm als Gottes Wort zur Erkenntnis kam. Zur Fähigkeit des Menschen, solches wahrzunehmen, gehört der Transzendenzbezug, den solche Wahrnehmungen mit sich bringen. Und im Transzendenzbezug entstehen neue Dimensionen der Lebendigkeit. Bei Paulus erscheint der Transzendenzbezug als Bereicherung einer Lebendigkeit, die im Gottesbezug geschieht: "Niemand von uns lebt in Bezug auf

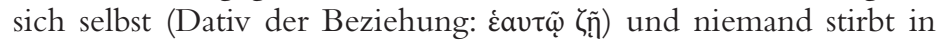
Bezug auf sich selbst. Wenn wir leben, leben wir dem Herrn, wenn wir sterben, sterben wir dem Herrn." (Röm 14,7.8a). ${ }^{24}$ Die eigene Lebendigkeit erhält eine neue Dimension dadurch, dass sie im Horizont des Christus geschieht. Weil es den Christus gibt und seine Art, die Menschen zu betrachten, ist die Selbstbezogenheit nicht mehr das einzig Massgebende. Im Horizont des Christus kommt der Mensch in einen neuen Seins-Raum (konkret erlebbar beispielsweise, wenn er einen Kirchenraum betritt): Es muss sich jetzt nicht mehr allein mit seinen eigenen Augen ansehen, sondern er kann sich mit den Augen des Christus neu betrachten. Im Raum einer Kirche kommt ein Mensch anders zur Sprache als im Raum seiner Selbstgespräche. Er ist nicht mehr beschränkt auf die unbarmherzige Sprache, die in derWelt der Konkurrenz gesprochen wird; er kommt in einen Sprachraum, der durch eine gnädige Sprache bestimmt ist, eine Sprache, die nichts beschönigt und dennoch den Menschen aus dem Bann seiner Taten und Untaten befreit.

ZurWahrnehmung gehört - um ein zweites Moment zu nennen die Möglichkeit, Beziehungen einzugehen. Weil Wahrnehmung zur Lebendigkeit gehört, gehören auch mannigfaltige Beziehungen zu ihr. Aus der Perspektive des Neuen Testaments könnte man sagen, dass Beziehungsreichtum fast ein Synonym für Lebendigkeit ist. Denn Lebendigkeit findet konkret in der Gestalt des $\sigma \tilde{\omega} \mu \alpha$, des Leibes, statt. Dabei ist der Leib nicht etwa ein Teil des Menschen neben anderen Teilen. Man kann nicht sagen, der Mensch habe einen Leib. Insbesondere nach Paulus gilt vielmehr, dass der Mensch ein $\sigma \tilde{\omega} \mu \alpha$

\footnotetext{
${ }^{24}$ Die bisweilen naheliegende ethische Auslegung des "Lebens für den Kyrios" ist hier schon dadurch ausgeschlossen, dass im Sterben von Ethik nicht mehr die Rede sein kann. Vielmehr geht es um bis in letzte Tiefen gedachte Zugehörigkeit zum Christus und insofern radikale Überwindung der Einsamkeit (mit Ulrich Wilckens, Der Brief an die Römer, 3. Teilband Röm 12-16 [1982], Zürich/Neukirchen-Vluyn 21988, 84).
} 
ist. ${ }^{25}$ Die Leiblichkeit wird sogar als Konstante wahrgenommen, die das irdische Leben mit dem Leben in himmlischerVollkommenheit verbindet: Einem $\sigma \tilde{\omega} \mu \alpha \psi v \cup$ เкóv, welches die gegenwärtige Existenz des Menschen charakterisiert, steht nach Paulus ein $\sigma \tilde{\omega} \mu \alpha \pi v \varepsilon v \mu \alpha \tau$ เ gegenüber, welches die Seinsweise im Eschaton beschreibt (1Kor 15,44). Der psychische Leib des Menschen wird im Eschaton auf vollendete Weise durch den göttlichen Geist definiert. Allerdings ist jener Geist schon jetzt eine Realität und entfaltet seine Wirkung schon in der psychischen Existenz des Menschen. Konkret besteht diese Wirkung in der Steigerung der Leiblichkeit beziehungsweise in der Befreiung des Menschen zu einer reichen Beziehung zu Gott, $\mathrm{zu}$ sich selbst und insofern auch $\mathrm{zu}$ den anderen Menschen und zur Welt. So sehr die Fähigkeit zur Wahrnehmung eine notwendige Bedingung für die Beziehungsdimension des Lebens ist, so sehr ist die Leiblichkeit, die irdisch durch Vergänglichkeit, Schwachheit und Niedrigkeit geprägt ist, darauf angewiesen, vom göttlichen Geist neu entdeckt und gesteigert $\mathrm{zu}$ werden. Auch in dieser Hinsicht versteht sich Lebendigkeit nicht von selbst, sie will von kreativen Kräften stets neu erschaffen werden. Im Transzendenzbezug kann der Mensch erfahren, dass es lebensfreundlich ist, sich jenen kreativen Kräften auszusetzen, welche die Lebendigkeit steigern, indem sie das Sein in Beziehungen fördern und so den Menschen von der Selbstbezogenheit zur Offenheit für Gott und die Welt bewegen.

\section{Zum Schluss}

Der theologische Umgang mit entscheidenden Charakteristika der natürlich gegebenen Lebendigkeit ist gekennzeichnet dadurch, dass jene Charakteristika rezipiert und als Bilder für die durch Gott geschaffene Lebendigkeit verstanden werden. Eigenschaften werden als Fragment aufgenommen, die eine Ahnung vom Ganzen zu geben vermögen. Im irdischen Fragment des Geschaffenen wird eine Kreativität wahrgenommen, die an den Schöpfer denken lässt. Dabei findet diese Rezeption metaphorisch statt, also so, dass die Differenz zwischen dem Fragment und dem Ganzen, zwischen dem Versprechen und dem Versprochenen stets im Bewusstsein bleibt und

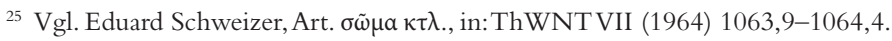


mitgesagt wird. Dabei erfahren die Fragmente eine Eindeutigkeit, die nicht von dieser Welt ist und die sie in dieser Welt auch nicht haben.

- Prof. em. Dr. Hans Weder, war von 1980-2000 ord. Professor für Neutestamentliche Wissenschaft, von 2000-2008 war er Rektor der Universität Zürich. Zu seinen Spezialgebieten gehören u.a. die Gleichnisse Jesu, die paulinische Kreuzestheologie und die neutestamentliche Hermeneutik. 